\title{
On the existence and uniqueness of solutions of the configurational probability diffusion equation for the generalized rigid dumbbell polymer model
}

\author{
Ionel S. Ciupercă and Liviu I. Palade
}

Communicated by Tudor Ratiu, received July 1, 2010.

\begin{abstract}
Kinetic phase-space theories (see [3]) have long been associated with successfully predicting the rheological properties of a variety of macromolecular fluids. Their cornerstone is the configurational probability density, essential to calculating the stress tensor. This function is a solution to the probability diffusion equation. In Section 2 we prove the existence and uniqueness of solutions to the corresponding evolutionary diffusion equation, in Section 3 to the stationary (time independent) equation; these problems, within the context of polymer dynamics theory, did not receive attention until now.
\end{abstract}

\section{CONTEnTs}

1. Introduction

2. Evolutionary boundary value problems 246

3. Stationary boundary value problems 257

4. Final comments 262

5. Acknowledgements 262

$\begin{array}{ll}\text { References } & 262\end{array}$

\section{Introduction}

The macroscopic flow behavior of elastic liquids is strongly related to the fluid microscopic architecture and molecular interactions. This has been recognized since the early days of modern rheology. Consequently, scientists took on to obtaining constitutive relationships relating the stress tensor to molecular complexity. A very successful theory dealing with this difficult task is the kinetic (phase-space) one developed by Bird, Curtiss, Armstrong and Hassager and their collaborators in [3]

2000 Mathematics Subject Classification. 35, 76.

Key words and phrases. phase-space kinetic theory, rigid dumbbell chains, Fokker-PlanckSmoluchowski configurational probability equation, existence and uniqueness of solutions. 
(for some early kinetic model fundamentals see Kirkwood's [9]; for other accounts on this topic and related molecular theories see e.g. $[\mathbf{1}],[\mathbf{8}],[\mathbf{1 0}],[\mathbf{1 1}],[\mathbf{1 2}],[\mathbf{1 3}]$, $[\mathbf{1 4}]$ and [15]). It has been applied to model and predict rheological properties of both polymer solutions and undiluted (including mixtures of) polymers.

The polymer chains are modeled either as spring-bead or rod-bead mechanical systems subjected to hydrodynamic, Brownian, intra-molecular interactions in the phase space. Statistical mechanics techniques (averages and projections) reduce the problem of the multi-chain liquid to the configuration space of a single macromolecule.

One of the salient ingredients in the polymer mean-field theories is the configuration (probability) distribution function, with the help of which the stress tensor is calculated. This function is actually the solution to diffusion equations (see equation 19.3-26 on page 322 and equation 19.5 -1 on page 328 in [3]) pertaining to the (large) family of Fokker-Planck-Smoluchowski partial differential equations (PDEs). In [3] series expansion solutions are obtained.

We undertake to proving the existence and uniqueness of solutions to the forementioned PDEs obeying physically meaningful initial boundary conditions, an issue that has not been addressed as yet. This work consists of two parts. Section 2 is devoted to the evolutionary (i.e. time dependent) boundary value problem and a procedure based on the Galerkin approximation is used. In Section 3 we prove the existence, uniqueness and positivity of a stationary solution. The proof uses the Krein-Rutman theory (see also [5] and [4] for related works on a diluted polymer FENE model and a neuroscience model respectively).

\section{Evolutionary boundary value problems}

2.1. The boundary value problems. The problems we study describe the configurational dynamics of polymer melts made up of Kramers chains. Specifically, each macromolecule is assimilated to a finite succession of freely jointed rods; the orientation of each rod is given by the unitary vector $x \in \mathbb{R}^{3}$. The macromolecule mass is concentrated on "beads" located at the joints. For full details see Chap. 19 in $[3]$.

Let the unit sphere be denoted $S_{2}:=\left\{x \in \mathbb{R}^{3}\right.$ s.t. $\left.\|x\|_{2}=1\right\}$. In the following we denote by " $\nabla$ " the gradient on the sphere $S_{2}$.

The first boundary value problem (referred to subsequently as P-1) reads:

Problem 1

Find $\tilde{\psi}: S_{2} \times[0,+\infty[\rightarrow \mathbb{R}$, with $\tilde{\psi}(x, t)$ solution to

$$
\begin{aligned}
& \frac{\partial \tilde{\psi}}{\partial t}-\alpha \nabla \cdot(\nabla \tilde{\psi})+\nabla \cdot(\tilde{a}(x) \tilde{\psi})=0, x \in S_{2}, t>0 \\
& \tilde{\psi} \geq 0 \\
& \int_{S_{2}} \tilde{\psi}(x, t) \mathrm{d} x=1, \forall t \geq 0 \\
& \tilde{\psi}(x, 0)=\tilde{\psi}_{0}(x)
\end{aligned}
$$

where $\alpha>0$ is a given, fluid related, parameter, and $\tilde{a}: \mathbb{R}^{3} \rightarrow \mathbb{R}^{3}, \tilde{\psi}_{0}: S_{2} \rightarrow \mathbb{R}$, $\tilde{\psi}_{0} \geq 0$, are known functions (the later being an initial - or equilibrium - probability distribution). Moreover, $\int_{S_{2}} \tilde{\psi}_{0}(x) \mathrm{d} x=1$. With our notations, this problem is 
basically equation $19.5-1$ on page 328 in [3].

The second boundary value problem (referred to subsequently as P-2) reads:

\section{Problem 2}

Find $\left.\tilde{\psi}: S_{2} \times\right] 0,1[\times[0,+\infty[\rightarrow \mathbb{R}$, with $\tilde{\psi}(x, \sigma, t)$ solution to

$$
\begin{aligned}
& \left.\frac{\partial \tilde{\psi}}{\partial t}-\alpha_{1} \frac{\partial^{2} \tilde{\psi}}{\partial \sigma^{2}}-\alpha_{2} \nabla \cdot(\nabla \tilde{\psi})+\nabla \cdot(\tilde{a}(x) \tilde{\psi})=0, x \in S_{2}, \sigma \in\right] 0,1[, t>0 \\
& \tilde{\psi} \geq 0 \\
& \left.\int_{S_{2}} \tilde{\psi}(x, \sigma, t) \mathrm{d} x=1, \forall \sigma \in\right] 0,1[, \forall t>0 \\
& \left.\tilde{\psi}\right|_{\sigma \in\{0,1\}}=\frac{1}{4 \pi} \\
& \left.\tilde{\psi}(x, \sigma, 0)=\tilde{\psi}_{0}^{\sigma}(x, \sigma), \forall x \in S_{2}, \forall \sigma \in\right] 0,1[
\end{aligned}
$$

where $\alpha_{1}, \alpha_{2}>0$ are given, fluid related, parameters and $\tilde{a}: \mathbb{R}^{3} \rightarrow \mathbb{R}^{3}$ is a known function. Next, $\left.\tilde{\psi}_{0}^{\sigma}: S_{2} \times\right] 0,1\left[\rightarrow \mathbb{R}, \tilde{\psi}_{0}^{\sigma} \geq 0\right.$, is the known initial configurational probability; it is such that $\left.\int_{\Omega_{2}} \tilde{\psi}_{0}^{\sigma}(x) \mathrm{d} x=1, \forall \sigma \in\right] 0,1[$. With our notations, this problem is basically equation $19.3-26$ on page 322 in [3].

In the following, for both P-1 and P-2, we assume that the following general hypothesis holds true:

$(\mathrm{H}): \tilde{a} \in \mathscr{C}^{\infty}(V)$ and $\tilde{a} \cdot x=0$ for all $x \in S_{2}$, where $V \subset \mathbb{R}^{3}$ is a neighborhood of $S_{2}$.

We shall make use of the short hand notation $x x=x \otimes x$. As customary in engineering/applied mathematics literature, a dot "." stands for summation over one repeated (dummy) index, ": " for summation over two repeated indices. For rigid dumbbell molecules, $\tilde{a}=A \cdot x-A: x x x$, where $A$ is a $3 \times 3$ matrix, henceforth compliance with hypothesis $(\mathrm{H})$ is ensured.

From [2] we observe, in the case of spherical coordinates, that:

$$
\begin{aligned}
& \nabla:=e_{\theta} \frac{\partial}{\partial \theta}+e_{\phi} \frac{1}{\sin \theta} \frac{\partial}{\partial \phi} \\
& \nabla \cdot \nabla=\triangle:=\frac{1}{\sin \theta} \frac{\partial}{\partial \theta}\left(\sin \theta \frac{\partial}{\partial \theta}\right)+\frac{1}{\sin ^{2} \theta} \frac{\partial^{2}}{\partial \phi^{2}}
\end{aligned}
$$

Moreover, for any vector $\tilde{b}$ such that $\tilde{b} \cdot x=0$, denoting $b_{1}=\tilde{b} \cdot e_{\theta}$ and $b_{2}=\tilde{b} \cdot e_{\phi}$, one has

$$
\nabla \cdot(\tilde{b} f)=\frac{1}{\sin \theta} \frac{\partial}{\partial \theta}\left(b_{1} f \sin \theta\right)+\frac{1}{\sin \theta} \frac{\partial}{\partial \phi}\left(b_{2} f\right)
$$

Let us now denote

$$
D=] 0, \pi[\times] 0,2 \pi[
$$

and

$$
\left.D_{\sigma}=D \times\right] 0,1[.
$$

Making use of the above results, one may now re-state problems $\mathrm{P}-1$ and $\mathrm{P}-2$ in the following way:

\section{Problem 1}


Search for solutions $\psi: D \times[0,+\infty[\rightarrow \mathbb{R}$ to the following initial-boundary value problem:

$$
\begin{array}{ll} 
& \frac{\partial \psi}{\partial t}-\alpha\left[\frac{1}{\sin \theta} \frac{\partial}{\partial \theta}\left(\sin \theta \frac{\partial \psi}{\partial \theta}\right)+\frac{1}{\sin ^{2} \theta} \frac{\partial^{2} \psi}{\partial \phi^{2}}\right]+\frac{1}{\sin \theta} \frac{\partial}{\partial \theta}\left(a_{1} \sin \theta \psi\right) \\
(2.10) & +\frac{1}{\sin \theta} \frac{\partial}{\partial \phi}\left(a_{2} \psi\right)=0,(\theta, \phi) \in D, t>0 \\
(2.11) & \psi \text { is a } \phi-\text { periodic function, of period } 2 \pi \\
(2.12) & \psi \geq 0 \\
(2.13) & \int_{D} \psi \sin \theta \mathrm{d} \theta \mathrm{d} \phi=1, \forall t>0 \\
(2.14) & \psi(\theta, \phi, 0)=\psi_{0}(\theta, \phi)
\end{array}
$$$$
\text { (2.13) } \quad \int_{D} \psi \sin \theta \mathrm{d} \theta \mathrm{d} \phi=1, \forall t>0
$$

where $\psi_{0}$ satisfies

$$
\psi_{0} \geq 0, \quad \int_{D} \psi_{0} \sin \theta \mathrm{d} \theta \mathrm{d} \phi=1
$$

\section{Problem 2}

Search for solutions $\psi: D_{\sigma} \times[0,+\infty[\rightarrow \mathbb{R}$ to the following initial-boundary value problem:

$$
\begin{aligned}
& \frac{\partial \psi}{\partial t}-\alpha_{1} \frac{\partial^{2} \psi}{\partial \sigma^{2}}-\alpha_{2}\left[\frac{1}{\sin \theta} \frac{\partial}{\partial \theta}\left(\sin \theta \frac{\partial \psi}{\partial \theta}\right)+\frac{1}{\sin ^{2} \theta} \frac{\partial^{2} \psi}{\partial \phi^{2}}\right] \\
& +\frac{1}{\sin \theta} \frac{\partial}{\partial \theta}\left(a_{1} \sin \theta \psi\right) \\
& +\frac{1}{\sin \theta} \frac{\partial}{\partial \phi}\left(a_{2} \psi\right)=0,(\theta, \phi) \in D, t>0 \\
& \psi \text { is a } \phi-\text { periodic function, of period } 2 \pi \\
& \psi \geq 0 \\
& \left.\int_{D} \psi \sin \theta \mathrm{d} \theta \mathrm{d} \phi=1, \forall t>0, \forall \sigma \in\right] 0,1[ \\
& \left.\psi\right|_{\sigma \in\{0,1\}}=\frac{1}{4 \pi} \\
& \psi(\theta, \phi, t=0)=\psi_{0}^{\sigma}(\theta, \phi, \sigma)
\end{aligned}
$$

where $\psi_{0}^{\sigma}$ satisfies

$$
\psi_{0} \geq 0, \quad \int_{D} \psi_{0} \sin \theta \mathrm{d} \theta \mathrm{d} \phi=1
$$

In the above we made use of the following notations: $a_{1}=a \cdot e_{\theta}, a_{2}=a \cdot e_{\phi}$.

\subsection{Functional spaces framework.}

2.2.1. Functional spaces for problem P-1. Let the following Hilbert spaces:

$$
L_{s}^{2}(D):=\left\{v \in L_{\mathrm{loc}}^{1}(D) \text { s.t. } \int_{D} v^{2} \sin \theta \mathrm{d} \theta \mathrm{d} \phi<\infty\right\}
$$




$$
\begin{aligned}
H_{s}^{1}(D):= & \left\{v \in L_{\mathrm{loc}}^{1}(D) \text { s.t. } v \text { periodic w.r.t. } \phi,\right. \\
& \left.\int_{D}\left(\sin \theta\left|\frac{\partial v}{\partial \theta}\right|^{2}+\frac{1}{\sin \theta}\left|\frac{\partial v}{\partial \phi}\right|^{2}+v^{2} \sin \theta\right) \mathrm{d} \theta \mathrm{d} \phi<\infty\right\}
\end{aligned}
$$

Notice the continuous, dense inclusions:

$$
H_{s}^{1}(D) \underset{\text { cont. }}{\subset} L_{s}^{2}(D)=\left(L_{s}^{2}(D)\right)^{\prime} \underset{\text { cont. }}{\subset}\left(H_{s}^{1}(D)\right)^{\prime} .
$$

Let now the linear operators be defined as following: $A_{1}: H_{s}^{1}(D) \rightarrow\left(H_{s}^{1}(D)\right)^{\prime}$, such that $\left\langle A_{1} u, v\right\rangle=\alpha \int_{D}\left(\sin \theta \frac{\partial u}{\partial \theta} \frac{\partial v}{\partial \theta}+\frac{1}{\sin \theta} \frac{\partial u}{\partial \phi} \frac{\partial v}{\partial \phi}\right) \mathrm{d} \theta \mathrm{d} \phi, \forall u, v \in H_{s}^{1}$, and $B_{1}$ : $L_{s}^{2}(D) \rightarrow\left(H_{s}^{1}(D)\right)^{\prime}$, such that $\left\langle B_{1} u, v\right\rangle=-\int_{D}\left(\sin \theta u \frac{\partial v}{\partial \theta}+a_{2} u \frac{\partial v}{\partial \phi}\right) \mathrm{d} \theta \mathrm{d} \phi$. Then, multiplying formally $(2.10)$ by $v \sin \theta$, with $v$ arbitrary in $H_{s}^{1}(D)$, and integrating on $D$ we deduce that $\mathrm{P}-1$ can be written in variational form as

$$
\begin{gathered}
\frac{d \psi}{d t}+A_{1} \psi+B_{1} \psi=0 \\
\psi(t=0)=\psi_{0}
\end{gathered}
$$

In the above, $\psi$ must be positive, $\psi \geq 0$, and such that $\int_{D} \psi \sin \theta \mathrm{d} \theta \mathrm{d} \phi=1$.

2.2.2. Functional spaces for problem P-2. Let the following Hilbert spaces $L_{s}^{2}\left(D_{\sigma}\right)$ and $H_{s 0}^{2}\left(D_{\sigma}\right)$ be defined as (with $\mathrm{d} D_{\sigma}=\mathrm{d} \theta \mathrm{d} \phi \mathrm{d} \sigma$ )

$$
\begin{aligned}
& L_{s}^{2}\left(D_{\sigma}\right):=\left\{v \in L_{\mathrm{loc}}^{1}\left(D_{\sigma}\right) \text { s.t. } \int_{D_{\sigma}} v^{2} \sin \theta \mathrm{d} D_{\sigma}<\infty\right\} \\
& H_{s 0}^{1}\left(D_{\sigma}\right):=\left\{v \in L_{\mathrm{loc}}^{1}\left(D_{\sigma}\right) \text { s.t. } v \text { periodic w.r.t. } \phi, v(\sigma=0)=v(\sigma=1)=0,\right. \\
& \left.\int_{D_{\sigma}}\left[\sin \theta\left(\frac{\partial v}{\partial \sigma}\right)^{2}+\sin \theta\left(\frac{\partial v}{\partial \theta}\right)^{2}+\frac{1}{\sin \theta}\left(\frac{\partial v}{\partial \phi}\right)^{2}+v^{2} \sin \theta\right] \mathrm{d} D_{\sigma}<\infty\right\}
\end{aligned}
$$

Notice the continuous, dense inclusions:

$$
H_{s 0}^{1}\left(D_{\sigma}\right) \underset{\text { cont. }}{\subset} L_{s}^{2}\left(D_{\sigma}\right)=\left(L_{s}^{2}\left(D_{\sigma}\right)\right)^{\prime} \underset{\text { cont. }}{\subset}\left(H_{s 0}^{1}\left(D_{\sigma}\right)\right)^{\prime} .
$$

Let now the linear operators be defined as following:

$$
A_{2}: H_{s 0}^{1}\left(D_{\sigma}\right) \rightarrow\left(H_{s 0}^{1}\left(D_{\sigma}\right)\right)^{\prime},
$$

such that

$$
\left\langle A_{2} u, v\right\rangle=\alpha_{1} \int_{D_{\sigma}}\left(\sin \theta \frac{\partial u}{\partial \theta} \frac{\partial v}{\partial \theta}+\frac{1}{\sin \theta} \frac{\partial u}{\partial \phi} \frac{\partial v}{\partial \phi}\right) \mathrm{d} D_{\sigma}+\alpha_{2} \int_{D_{\sigma}} \sin \theta \frac{\partial u}{\partial \sigma} \frac{\partial v}{\partial \sigma} \mathrm{d} D_{\sigma},
$$

and

such that

$$
B_{2}: L_{s}^{2}\left(D_{\sigma}\right) \rightarrow\left(H_{s 0}^{1}\left(D_{\sigma}\right)\right)^{\prime},
$$

$$
\left\langle B_{2} u, v\right\rangle=-\int_{D_{\sigma}}\left(\sin \theta u \frac{\partial v}{\partial \theta}+a_{2} u \frac{\partial v}{\partial \phi}\right) \mathrm{d} \theta \mathrm{d} \phi
$$

Next, let 


$$
\psi=\frac{1}{4 \pi}+\bar{\psi}
$$

Then, P-2 can be stated as: find $\bar{\psi}:\left[0,+\infty\left[\rightarrow H_{s 0}^{1}\left(D_{\sigma}\right)\right.\right.$ solution to the variational problem expressed as

$$
\begin{gathered}
\frac{d \bar{\psi}}{d t}+A_{2} \bar{\psi}+B_{2} \bar{\psi}=f \\
\bar{\psi}(t=0)=\psi_{0}^{\sigma}-\frac{1}{4 \pi} \\
\frac{1}{4 \pi}+\bar{\psi} \geq 0 \\
\int_{D} \bar{\psi} \sin \theta \mathrm{d} \theta \mathrm{d} \phi=0, \forall \sigma \in[0,1], \forall t
\end{gathered}
$$

where $\langle f, v\rangle=\frac{1}{4 \pi} \int_{D}\left(a_{1} \sin \theta \frac{\partial v}{\partial \theta}+a_{2} \frac{\partial v}{\partial \phi}\right) \mathrm{d} D_{\sigma}, \forall v \in H_{s 0}^{1}\left(D_{\sigma}\right)$. Moreover, with $r, \theta, \phi$ being the spherical coordinates of a position vector $x$, denote

$$
\Omega:=\left\{x \in \mathbb{R}^{3}, 1<r<2\right\},
$$

and $\Omega_{\sigma}$ the 4-dimensional domain

$$
\Omega_{\sigma}:=\left\{(x, \sigma) \in \mathbb{R}^{3} \times\right] 0,1[, 1<r<2\},
$$

and

$$
V_{\sigma}:=\left\{u \in H^{1}\left(\Omega_{\sigma}\right), u(\sigma=0)=u(\sigma=1)=0\right\} .
$$

REMARK 2.1. Any function $u(\theta, \Phi)$ defined in $D$ can be viewed as a function defined in $\Omega$ independent on $r$.

Let us now prove the first result of this Section.

Proposition 2.1. (i) $u \in L_{s}^{2}(D) \Leftrightarrow u \in L^{2}(\Omega)$ and $u$ independent of $r$; moreover

$\|u\|_{L_{s}^{2}(D)} \leq\|u\|_{L^{2}(\Omega)} \leq 2\|u\|_{L_{s}^{2}(D)}$.

(ii) $u \in H_{s}^{1}(D) \Leftrightarrow u \in H^{1}(\Omega)$ and $u$ independent of $r$; moreover

$\frac{1}{2}\|u\|_{H_{s}^{1}(D)} \leq\|u\|_{H^{1}(\Omega)} \leq 2\|u\|_{H^{1}(\Omega)}$.

(iii) $u \in L_{s}^{2}\left(D_{\sigma}\right) \Leftrightarrow u \in L^{2}\left(\Omega_{\sigma}\right)$ and $u$ independent of $r$; moreover

$\|u\|_{L^{2}\left(D_{\sigma}\right)} \leq\|u\|_{L^{2}\left(\Omega_{\sigma}\right)} \leq 2\|u\|_{L^{2}\left(D_{\sigma}\right)}$.

(iv) $u \in H_{s 0}^{1}\left(D_{\sigma}\right) \Leftrightarrow u \in V_{\sigma}$ and $u$ independent of $r$; moreover

$\frac{1}{2}\|u\|_{H_{s 0}^{1}\left(D_{\sigma}\right)} \leq\|u\|_{H^{1}\left(\Omega_{\sigma}\right)} \leq 2\|u\|_{H_{s 0}^{1}\left(D_{\sigma}\right)}$.

Proof. The proof of the above statements is a direct consequence of the following formulae

$$
\begin{aligned}
\|u\|_{L^{2}(\Omega)}^{2} & =\int_{D} \int_{0}^{1}|u|^{2} r^{2} \sin \theta \mathrm{d} r \mathrm{~d} \theta \mathrm{d} \phi \\
\|u\|_{H^{1}(\Omega)}^{2} & =\int_{D} \int_{0}^{1}\left[\frac{1}{r^{2}}\left(\frac{\partial u}{\partial \theta}\right)^{2}+\frac{1}{r^{2} \sin ^{2} \theta}\left(\frac{\partial u}{\partial \phi}\right)^{2}+u^{2}\right] r^{2} \sin \theta \mathrm{d} r \mathrm{~d} \theta \mathrm{d} \phi
\end{aligned}
$$




$$
\begin{aligned}
& \|u\|_{L^{2}\left(\Omega_{\sigma}\right)}^{2}=\int_{D} \int_{0}^{1} \int_{0}^{1}|u|^{2} r^{2} \sin \theta \mathrm{d} r \mathrm{~d} \theta \mathrm{d} \phi \mathrm{d} \sigma \\
& \|u\|_{H^{1}\left(\Omega_{\sigma}\right)}^{2}=\int_{D} \int_{0}^{1} \int_{0}^{1}\left[\left(\frac{\partial u}{\partial \sigma}\right)^{2}+\frac{1}{r^{2}}\left(\frac{\partial u}{\partial \theta}\right)^{2}+\frac{1}{r^{2} \sin ^{2} \theta}\left(\frac{\partial u}{\partial \phi}\right)^{2}+u^{2}\right] \\
& r^{2} \sin \theta \mathrm{d} r \mathrm{~d} \theta \mathrm{d} \phi \mathrm{d} \sigma
\end{aligned}
$$

Before ending this Section, we prove the following:

Proposition 2.2. The inclusions $H_{s}^{1}(D) \subset L_{s}^{2}(D)$ and $H_{s 0}^{1}\left(D_{\sigma}\right) \subset L_{s}^{2}\left(D_{\sigma}\right)$ are compact.

Proof. We focus on proving the first inclusion as the proof for the second one is similar in nature.

Let $u_{n}$ be a bounded sequence of elements of $H_{s}^{1}(D)$, then $u_{n}$ is bounded in $H^{1}(\Omega)$. Invoking the compact inclusion $H^{1}(\Omega) \subset L^{2}(\Omega)$, we get the existence of an element $u \in H^{1}(\Omega)$ and of a sub-sequence of $u_{n}$ - for convenience also denoted $u_{n}$ such that $u_{n} \underset{n \rightarrow \infty}{\rightarrow} u$ in $H^{1}(\Omega)$, and $u_{n} \underset{n \rightarrow \infty}{\longrightarrow} u$ in $L^{2}(\Omega)$. Moreover $\frac{\partial u_{n}}{\partial r} \underset{n \rightarrow \infty}{\rightarrow} \frac{\partial u}{\partial r}$ in $L^{2}(\Omega)$. As $\frac{\partial u_{n}}{\partial r}=0$, this gives $\frac{\partial u}{\partial r}=0$, henceforth $u=u(\theta, \phi)$. Then $u \in L_{s}^{2}(D)$ and $u_{n} \underset{n \rightarrow \infty}{\longrightarrow} u$ in $L^{2}(\Omega)$.

2.3. Evolutionary boundary value problems: existence and uniqueness results. Observe first that for any $\beta>0$ the operator $A_{1}+\beta$ Id is invertible and its inverse maps $\left(H_{s}^{1}(D)\right)^{\prime}$ onto $H_{s}^{1}(D)$.

As the inclusion $H_{s}^{1}(D) \subset L_{s}^{2}(D)$ is compact, one deduces the existence of a sequence of eigenvectors of $A_{1}$, i.e. $0 \leq \lambda_{1}^{1} \leq \lambda_{2}^{1} \leq \cdots \leq \lambda_{k}^{1} \leq \cdots \rightarrow+\infty$, and that of a orthonormal basis for the space $L_{s}^{2}(D)$ consisting of eigenvectors $\varphi_{1}^{1}, \varphi_{2}^{1} \ldots \varphi_{k}^{1} \ldots$ Invoking arguments similar in nature one deduces the existence of a sequence of eigenvectors of $A_{2}$, i.e. $0 \leq \lambda_{1}^{2} \leq \lambda_{2}^{2} \leq \cdots \leq \lambda_{k}^{2} \leq \cdots \rightarrow+\infty$, and that of a orthonormal basis for the space $L_{s}^{2}\left(D_{\sigma}\right)$ consisting of eigenvectors $\varphi_{1}^{2}, \varphi_{2}^{2} \ldots \varphi_{k}^{2} \ldots$

One has the following result:

THEOREM 2.1. Let $\psi_{0} \in L_{s}^{2}(D), T>0$. There exists a unique solution $\psi$ to equations (2.23)-(2.24), s.t. $\psi \in L^{2}\left(0, T ; H_{s}^{1}(D)\right) \cap \mathscr{C}^{0}\left(0, T ; L_{s}^{2}(D)\right)$ and $\frac{d \psi}{d t} \in$ $L^{2}\left(0, T ;\left(H_{s}^{1}(D)\right)^{\prime}\right)$. In addition, whenever $\psi_{0}$ obeys $(2.15), \psi$ also solves $(2.12)$ (2.13).

Proof. Multiply $(2.10)$ by $\psi \sin (\theta)$ and integrate on $D$. It entails:

$$
\begin{aligned}
& \frac{1}{2} \frac{d}{d t}\|\psi\|_{L_{s}^{2}}^{2}+\alpha \int_{D}\left[\left(\frac{\partial \psi}{\partial \theta}\right)^{2} \sin \theta+\frac{1}{\sin \theta}\left(\frac{\partial \psi}{\partial \phi}\right)^{2}\right] \mathrm{d} \theta \mathrm{d} \phi \\
& -\int_{D}\left(a_{1} \psi \frac{\partial \psi}{\partial \theta} \sin \theta+a_{2} \psi \frac{\partial \psi}{\partial \phi}\right) \mathrm{d} \theta \mathrm{d} \phi=0
\end{aligned}
$$

Since: 


$$
\begin{gathered}
\int_{D} a_{1} \psi \frac{\partial \psi}{\partial \theta} \sin \theta \mathrm{d} \theta \mathrm{d} \phi \leq \frac{\alpha}{2} \int_{D}\left(\frac{\partial \psi}{\partial \theta}\right)^{2} \sin \theta \mathrm{d} \theta \mathrm{d} \phi+\frac{1}{2 \alpha} \int_{D} a_{1}^{2} \psi^{2} \sin \theta \mathrm{d} \theta \mathrm{d} \phi \\
\int_{D} a_{2} \psi \frac{\partial \psi}{\partial \phi} \mathrm{d} \theta \mathrm{d} \phi \leq \frac{\alpha}{2} \int_{D} \frac{1}{\sin \theta}\left(\frac{\partial \psi}{\partial \phi}\right)^{2} \mathrm{~d} \theta \mathrm{d} \phi+\frac{1}{2 \alpha} \int_{D} a_{2}^{2} \psi^{2} \sin \theta \mathrm{d} \theta \mathrm{d} \phi
\end{gathered}
$$

as $a_{1}, a_{2} \in L^{\infty}(D)$, the above estimates lead to:

$$
\frac{d}{d t}\|\psi\|_{L_{s}^{2}}^{2}+\alpha\|\psi\|_{H_{s}^{1}}^{2} \leq c\|\psi\|_{L_{s}^{2}}^{2}
$$

where $c>0$. Further on, making use of Gronwall's inequality, one gets:

$$
\begin{gathered}
\|\psi(t)\|_{L_{s}^{2}} \leq\left\|\psi_{0}\right\|_{L_{s}^{2}} e^{c T / 2}, \quad \forall t \in[0, T] \\
\|\psi(t)\|_{L^{2}\left(0, T ; H_{s}^{1}\right)} \leq \frac{1}{\alpha}\left(\left\|\psi_{0}\right\|_{L_{s}^{2}}^{2}+c T\left\|\psi_{0}\right\|_{L_{s}^{2}}^{2} e^{c T}\right)
\end{gathered}
$$

The rest of the proof is classical in the sense we use the Galerkin's method to obtain an approximate solution $\psi^{n}=\sum_{k=1}^{n} \gamma_{k}(t) \varphi_{k}^{1}$. Next, estimates similar in nature to those in equations (2.33)-(2.34) can be easily obtained. Letting $n \rightarrow+\infty$ leads to the result.

Taking now as test function $\varphi=1$ in $(2.23)$, invoking $\left\langle A_{1} \psi, 1\right\rangle=0,\left\langle B_{1} \psi, 1\right\rangle=$ 0 , leads to

$$
\frac{d}{d t} \int_{D} \psi \sin \theta \mathrm{d} \theta \mathrm{d} \phi=0
$$

from which one infers $\int_{D} \psi \sin \theta \mathrm{d} \theta \mathrm{d} \phi=\int_{D} \psi_{0} \sin \theta \mathrm{d} \theta \mathrm{d} \phi=1$.

Next, denote $\psi^{+}=\max \{\psi, 0\}, \psi^{-}=-\min \{\psi, 0\}, \psi=\psi^{+}-\psi^{-}$. Apply (2.23) to $\psi^{-}$. It gives $-\frac{1}{2} \frac{d}{d t}\left|\psi^{-}\right|^{2}-\left\langle A_{1} \psi^{-}, \psi^{-}\right\rangle+\left\langle B_{1} \psi^{-}, \psi^{-}\right\rangle=0$. We easily obtain (2.33) with $\psi^{-}$in the place of $\psi$. As $\psi_{0}^{-}=0$, it follows $\psi^{-}=0$, and hence $\psi \geq 0$.

For P-2 we have the following existence and uniqueness result:

Theorem 2.2. Let $\psi_{0} \in L_{s}^{2}\left(D_{\sigma}\right), T>0$. Then there exists a unique solution $\bar{\psi}$ to equations (2.25)-(2.26) s.t. $\bar{\psi} \in L^{2}\left(0, T ; H_{s 0}^{1}\left(D_{\sigma}\right)\right) \cap \mathscr{C}\left([0, T] ; L_{s}^{2}\left(D_{\sigma}\right)\right)$ and $\frac{d \bar{\psi}}{d t} \in L^{2}\left(0, T ;\left(H_{s 0}^{1}\left(D_{\sigma}\right)\right)^{\prime}\right)$. In addition, whenever $\psi_{0}^{\sigma}$ solves $(2.22), \bar{\psi}$ solves $(2.28)$ (2.29).

Proof. The proof of the existence and uniqueness result is similar to that of Theorem 2.1.

Let us now prove that $\psi \equiv \frac{1}{4 \pi}+\bar{\psi} \geq 0$. Applying (2.26) to

$$
\psi^{-} \in L^{2}\left(0, T ; H_{s 0}^{1}\left(D_{\sigma}\right)\right)
$$


we obtain

$$
\begin{aligned}
& \left\langle\frac{d \psi}{d t}, \psi^{-}\right\rangle+\alpha_{1} \int_{D_{\sigma}}\left(\frac{\partial \psi}{\partial \theta} \frac{\partial \psi^{-}}{\partial \theta} \sin \theta+\frac{1}{\sin \theta} \frac{\partial \psi}{\partial \phi} \frac{\partial \psi^{-}}{\partial \phi}\right) d D_{\sigma}+ \\
& \alpha_{2} \int_{D_{\sigma}} \frac{\partial \psi}{\partial \sigma} \frac{\partial \psi^{-}}{\partial \sigma} \sin \theta d D_{\sigma}-\int_{D_{\sigma}}\left(a_{1} \psi \frac{\partial \psi^{-}}{\partial \theta} \sin \theta+a_{2} \psi \frac{\partial \psi^{-}}{\partial \phi}\right) d D_{\sigma}=0 .
\end{aligned}
$$

The above leads, as in Theorem 2.1 , to $\psi^{-}=0$, that is $\psi \geq 0$.

In order to prove (2.29), consider in (2.26) an arbitrary test function $\varphi \in$ $H_{0}^{1}(] 0,1[)$, with $\varphi$ depending uniquely on $\sigma$. Denote $\tilde{\psi}:=\int_{D} \bar{\psi} \sin \theta \mathrm{d} \theta \mathrm{d} \phi$. Then $\left\langle\frac{d \bar{\psi}}{d t}, \varphi\right\rangle+\alpha_{2} \int_{0}^{1} \frac{\partial \bar{\psi}}{\partial \sigma} \frac{\partial \varphi}{\partial \sigma} \mathrm{d} \sigma=0$, where $\tilde{\psi} \in L^{2}\left(0, T ; H_{0}^{1}(] 0,1[)\right), \tilde{\psi}(t=0)=0$. This eventually gives $\tilde{\psi}=0$ and $(2.29)$ is proved.

2.4. Connection between the two probability configuration equations. Denote $\psi$ the solution to problem P-1, and $\psi_{\sigma}$ the solution to problem P-2. Let also $\psi_{\sigma}=\frac{1}{4 \pi}+\overline{\psi_{\sigma}}$. From now on we take $\alpha=\alpha_{2}$.

We shall prove in the following that $\overline{\psi_{\sigma}}$ can be Fourier expanded as following:

$$
\overline{\psi_{\sigma}}(t, \theta, \phi, \sigma)=\sum_{n=1}^{\infty} \sin (n \pi \sigma) g_{n}(t, \theta, \phi)
$$

where $g_{n}$ is to be found.

Observe that $1=\sum_{n=1}^{\infty} d_{n} \sin (n \pi \sigma)$, with $d_{n}=2 \int_{0}^{1} \sin (n \pi \sigma) \mathrm{d} \sigma$. Moreover, $d_{n}=0$ for $n$ even, and $d_{n}=\frac{4}{n \pi}$ for $n$ odd.

Let $\overline{\psi_{\sigma}^{n}}$ given by

$$
\overline{\psi_{\sigma}^{n}}=\sum_{k=1}^{n} \sin (k \pi \sigma) g_{k}(t, \theta, \phi)
$$

denote an approximation for $\overline{\psi_{\sigma}}$ for $n$ large enough, where $\overline{\psi_{\sigma}^{n}}$ solves

$$
\begin{gathered}
\frac{d}{d t} \overline{\psi_{\sigma}^{n}}+A_{2} \overline{\psi_{\sigma}^{n}}+B_{2} \overline{\psi_{\sigma}^{n}}=f \sum_{k=1}^{n} d_{k} \sin (k \pi \sigma) \\
\overline{\psi_{\sigma}^{n}}(t=0)=\sum_{k=1}^{n} g_{0 k} \sin (k \pi \sigma)
\end{gathered}
$$

where $g_{0 k}$ is given in equation (2.44) below.

Remark that

$$
A_{2} \overline{\psi_{\sigma}^{n}}=\alpha_{1} \sum_{k=1}^{n} k^{2} \pi^{2} \sin (k \pi \sigma) g_{k}(t, \theta, \phi)+\sum_{k=1}^{n} \sin (k \pi \sigma) A_{1}\left(g_{k}\right)
$$




$$
B_{2} \overline{\psi_{\sigma}^{n}}=\sum_{k=1}^{n} \sin (k \pi \sigma) B_{1}\left(g_{k}\right)
$$

From equations (2.38)-(2.41) above one concludes the $g_{k}$ functions are solutions to the following problem: find $g_{k} \in L^{2}\left(0, T ; H_{s}^{1}(D)\right) \cap \mathscr{C}^{0}\left([0, T] ; L_{s}^{2}(D)\right)$ s.t.

$$
\begin{gathered}
\frac{\partial g_{k}}{\partial t}+\alpha_{1} k^{2} \pi^{2} g_{k}+A_{1}\left(g_{k}\right)+B_{1}\left(g_{k}\right)=d_{k} f \\
g_{k}(0)=g_{0 k} \\
g_{0 k}=2 \int_{0}^{1}\left(\overline{\psi_{0}^{\sigma}}-\frac{1}{4 \pi}\right) \sin (k \pi \sigma) \mathrm{d} \sigma
\end{gathered}
$$

We now prove the following result:

Theorem 2.3. Let $T>0$. Assume the initial data $\overline{\psi_{0}^{\sigma}}$ is such that $\left\|g_{0 k}\right\|_{L_{s}^{2}} \leq$ $\frac{c}{k^{2}}$, with $c$ independent of $k$. Then $\overline{\psi_{\sigma}^{n}} \underset{n \rightarrow \infty}{\longrightarrow} \overline{\psi_{\sigma}}$ in

$$
L^{2}\left(0, T ; H_{s 0}^{1}\left(D_{\sigma}\right)\right) \cap L^{\infty}\left(0, T ; L_{s}^{2}\left(D_{\sigma}\right)\right) .
$$

Proof. Existence and uniqueness of solutions to the problem given in equations (2.42)-(2.43) is easily proved as in Theorem 2.1. Applying (2.42) to $g_{k}$ one gets:

$$
\frac{1}{2} \frac{d}{d t}\left\|g_{k}\right\|_{L_{s}^{2}}^{2}+\alpha_{1} k^{2} \pi^{2}\left\|g_{k}\right\|_{L_{s}^{2}}^{2}+\left\langle A_{1} g_{k}, g_{k}\right\rangle+\left\langle B_{1} g_{k}, g_{k}\right\rangle=d_{k}\left\langle f, g_{k}\right\rangle
$$

However, there exists $\gamma>0$ s.t.

$$
\left\langle A_{1} g_{k}, g_{k}\right\rangle+\left\langle B_{1} g_{k}, g_{k}\right\rangle+\gamma\left\|g_{k}\right\|_{L_{s}^{2}}^{2} \geq \alpha_{2}\left\|g_{k}\right\|_{H_{s}^{1}}^{2}
$$

from which one infers

$$
\frac{1}{2} \frac{d}{d t}\left\|g_{k}\right\|_{L_{s}^{2}}^{2}+\left(\alpha_{1} k^{2} \pi^{2}-\gamma\right)\left\|g_{k}\right\|_{L_{s}^{2}}^{2}+\alpha_{2}\left\|g_{k}\right\|_{H_{s}^{1}}^{2} \leq d_{k}\left\langle f, g_{k}\right\rangle
$$

Further on, using the result for $d_{n}$ previosuly obtained, we give for $k$ large enough:

$$
d_{k}\left\langle f, g_{k}\right\rangle \leq \frac{4}{k \pi}\|f\|_{L_{s}^{2}}^{2}\left\|g_{k}\right\|_{L_{s}^{2}}^{2} \leq \frac{1}{2}\left(\alpha_{1} k^{2} \pi^{2}-\gamma\right)\left\|g_{k}\right\|_{L_{s}^{2}}^{2}+\frac{8\|f\|_{L_{s}^{2}}^{2}}{2 k^{2} \pi^{2}\left(\alpha_{1} k^{2} \pi^{2}-\gamma\right)}
$$

From (2.47) one deduces there exists $c>0$ s.t.

$$
\frac{d}{d t}\left\|g_{k}\right\|_{L_{s}^{2}}^{2}+\left(\alpha_{1} k^{2} \pi^{2}-\gamma\right)\left\|g_{k}\right\|_{L_{s}^{2}}^{2}+2 \alpha_{2}\left\|g_{k}\right\|_{H_{s}^{1}}^{2} \leq \frac{c}{k^{4}}
$$

from which, upon integration from 0 to $T$, it gives 


$$
\begin{gathered}
\left\|g_{k}\right\|_{L^{\infty}\left(0, T ; L_{s}^{2}\right)}^{2}+\left\|g_{k}\right\|_{L^{2}\left(0, T ; H_{s}^{1}\right)}^{2} \leq \frac{c}{k^{4}} \\
\left\|g_{k}\right\|_{L^{\infty}\left(0, T ; L_{s}^{2}\right)}^{2} \leq \frac{c}{k^{6}}
\end{gathered}
$$

Next, from equation (2.37) one gets:

$$
\left\|\overline{\psi_{\sigma}^{n}}\right\|_{L_{s}^{2}\left(D_{\sigma}\right)}^{2}=\frac{1}{2} \int_{D_{\sigma}} \int_{0}^{1}\left|\sum_{k=1}^{n} \sin (k \pi \sigma) g_{k}(t, \theta, \phi)\right|^{2} \sin \theta \mathrm{d} \sigma \mathrm{d} \theta \mathrm{d} \phi=\frac{1}{2} \sum_{k=1}^{n}\left\|g_{k}\right\|_{L_{s}^{2}(D)}^{2}
$$

In a similar way one obtains that

$$
\left\|\frac{\partial}{\partial \sigma} \overline{\psi_{\sigma}^{n}}\right\|_{L_{s}^{2}\left(D_{\sigma}\right)}^{2}=\frac{\pi^{2}}{2} \sum_{k=1}^{n} k^{2}\left\|g_{k}\right\|_{L_{s}^{2}(D)}^{2}
$$

Therefore

$$
\left\|\overline{\psi_{\sigma}^{n}}\right\|_{H_{s 0}^{1}\left(D_{\sigma}\right)}^{2} \leq c \sum_{k=1}^{n} k^{2}\left\|g_{k}\right\|_{L_{s}^{2}(D)}^{2}
$$

As $\sum_{k=1}^{+\infty} \frac{1}{k^{2}}$ is convergent, invoking equations $(2.50)-(2.51)$ one observes that $\left\|\overline{\psi_{\sigma}^{n}}\right\|_{L^{\infty}\left(0, T ; L_{s}^{2}\left(D_{\sigma}\right)\right)}$ and $\left\|\overline{\psi_{\sigma}^{n}}\right\|_{L^{2}\left(0, T ; H_{s 0}^{1}\left(D_{\sigma}\right)\right)}$ are bounded and independent of $n$. Therefore, there exists $\xi \in L^{\infty}\left(0, T ; L_{s}^{2}\left(D_{\sigma}\right)\right) \cap L^{2}\left(0, T ; H_{s 0}^{1}\left(D_{\sigma}\right)\right)$ s.t. $\overline{\psi_{\sigma}^{n}} \underset{n \rightarrow+\infty}{\longrightarrow} \xi$ weakly.

Taking the limit $n \rightarrow+\infty$ in equation (2.38) and using the uniqueness of the solution to the problem P-2 leads to $\overline{\psi_{\sigma}}=\xi$.

One can now state that

$$
\overline{\psi_{\sigma}}=\sum_{k=1}^{\infty} g_{n} \sin (n \pi \sigma)
$$

Before ending this Section we shall establish several connections between this work results and those in Section 19.5 in $[\mathbf{3}]$.

Let us first notice that $f=-\frac{B_{1}(1)}{4 \pi}$. Let $\eta_{k}=g_{k}+\frac{d_{k}}{4 \pi}$. From equations (2.42)-(2.43) it follows that $\eta_{k}$ solves

$$
\begin{aligned}
& \frac{\partial \eta_{k}}{\partial t}+\alpha_{1} k^{2} \pi^{2}\left(\eta_{k}-\frac{d_{k}}{4 \pi}\right)+A_{1} \eta_{k}+B_{1} \eta_{k}=0 \\
& \eta_{k}(0)=g_{k 0}+\frac{d_{k}}{4 \pi}
\end{aligned}
$$

Therefore one has:

$$
\frac{\partial \eta_{k}}{\partial t}+\alpha_{1} k^{2} \pi^{2} \eta_{k}+A_{1} \eta_{k}+B_{1} \eta_{k}=\frac{\alpha_{1} k^{2} \pi d_{k}}{4}
$$




$$
\eta_{k}(0)=\eta_{0 k}=2 \int_{0}^{1} \psi_{0}^{\sigma} \sin (k \pi \sigma) \mathrm{d} \sigma
$$

Since $\sum_{k=1}^{\infty} \frac{d_{k}}{4 \pi} \sin (k \pi \sigma)=\frac{1}{4 \pi}$, by equation (2.55) one obtains

$$
\psi_{\sigma}=\sum_{n=1}^{\infty} \eta_{n} \sin (n \pi \sigma)
$$

We must now calculate $\eta_{k}$. To do so we first carry out the function change $z_{k}(t, \theta, \phi)=e^{\alpha_{1} k^{2} \pi^{2} t} \eta_{k}(t, \theta, \phi)$

which, after some calculations, gives

$$
\begin{gathered}
\frac{\partial z_{k}}{\partial t}+A_{1} z_{k}+B_{1} z_{k}=\frac{\alpha_{1} k^{2} \pi d_{k}}{4} e^{\alpha_{1} k^{2} \pi^{2} t} \\
z_{k}(t=0)=\eta_{0 k}
\end{gathered}
$$

The solution to the above given problem may be obtained using Duhamel's formula and written in the following form:

$$
z_{k}=e^{-\left(A_{1}+B_{1}\right) t} \eta_{0 k}+\int_{0}^{t} e^{-\left(A_{1}+B_{1}\right)\left(t-t^{\prime}\right)} e^{\alpha_{1} k^{2} \pi^{2} t^{\prime}} \frac{\alpha_{1} k^{2} \pi d_{k}}{4} \mathrm{~d} t^{\prime}
$$

Now $\eta_{k}$ can be calculated: assuming $\psi_{0}^{\sigma}=\frac{1}{4 \pi}$ (in which case $\eta_{0 k}=\frac{d_{k}}{4 \pi}$ ), it looks

$$
\eta_{k}=d_{k} e^{-\alpha_{1} k^{2} \pi^{2} t} F(u, t, 0)+\alpha_{1} k^{2} \pi^{2} d_{k} \int_{0}^{t} e^{-\alpha_{1} k^{2} \pi^{2}\left(t-t^{\prime}\right)} F\left(u, t, t^{\prime}\right) \mathrm{d} t^{\prime}
$$

where, in the above, $u$ stands for $(\theta, \phi)$, and $F\left(u, t, t^{\prime}\right)=\frac{1}{4 \pi} e^{-\left(A_{1}+B_{1}\right)\left(t-t^{\prime}\right)}$. $F\left(u, t, t^{\prime}\right)$ is a notation first employed on page 328 in [3].

Next, making use of equations (2.58) and (2.62) and using the fact that $d_{n}=0$ for $n$ even, leads to

$$
\psi_{\sigma}=F(u, t, 0) \sum_{\substack{n=1 \\ \text { n odd }}}^{\infty} e^{-\alpha_{1} n^{2} \pi^{2} t} d_{n} \sin (n \pi \sigma)+\int_{0}^{t} \tilde{P}\left(\sigma, t-t^{\prime}\right) F\left(u, t, t^{\prime}\right) \mathrm{d} t^{\prime}
$$

where

$$
\tilde{P}(\sigma, s)=\sum_{\substack{n=1 \\ \text { n odd }}}^{\infty} 4 \alpha_{1} n \pi e^{-\alpha_{1} n^{2} \pi^{2} s} \sin (n \pi \sigma)
$$


Notice the above $\tilde{P}$ is basically function $P$ obtained on page 328 in [3]. Also, taking $F(u, 0,0)=\frac{1}{4 \pi}$, from our equation (2.63) one gets

$$
\psi_{\sigma}(t=0)=F(u, 0,0) \sum_{\substack{n=1 \\ n \text { odd }}}^{\infty} d_{n} \sin (n \pi \sigma)=\frac{1}{4 \pi},
$$

as expected. Moreover, in this case $g_{0 k}=0$ (see equation (2.42)), and the constitutive assumption in Theorem 2.3 is obeyed.

\section{Stationary boundary value problems}

The corresponding stationary boundary value problems (referred to as SP-1 and SP-2) are:

Stationary Problem 1 (SP-1): Find $\psi_{s} \in H_{s}^{1}(D)$ that solves

$$
\psi_{s} \geq 0, \quad \int_{D} \psi_{s} \sin \theta \mathrm{d} \theta \mathrm{d} \phi=1
$$

Stationary Problem 2 (SP-2): Find $\overline{\psi_{s}} \in H_{s 0}^{1}(D)$ that solves

$$
\begin{gathered}
A_{2} \overline{\psi_{s}}+B_{2} \overline{\psi_{s}}=-\frac{1}{4 \pi} B_{2}(1) \\
\left.\frac{1}{4 \pi}+\overline{\psi_{s}} \geq 0, \quad \int_{D} \overline{\psi_{s}} \sin \theta \mathrm{d} \theta \mathrm{d} \phi=0, \quad \forall \sigma \in\right] 0,1[.
\end{gathered}
$$

For convenience we recall operators $A_{1,2}, B_{1,2}$ have been defined in Sections 2.2.1 and 2.2.2. Moreover, $r, \theta, \phi$ are the spherical coordinates of a position vector $x$; denote $\Omega:=\left\{x \in \mathbb{R}^{3}, 1<r<2\right\}, \Omega_{\sigma}$ the 4-dimensional domain $\Omega_{\sigma}:=$ $\left\{(x, \sigma) \in \mathbb{R}^{3} \times\right] 0,1[, 1<r<2\}$, and $V_{\sigma}:=\left\{u \in H^{1}\left(\Omega_{\sigma}\right), u(\sigma=0)=u(\sigma=1)=0\right\}$.

We solve the above presented problems using, in each case, a specific method.

It is clear that any function $u(\theta, \Phi)$ defined in $D$ can be viewed as a function defined in $\Omega$ independent on $r$.

3.1. Existence and uniqueness of solutions for the problem SP-1. Solving problem SP-1 boils down to proving $\lambda=0$ is an eigenvalue for the operator $A_{1}+B_{1}$. Use fo the Krein-Rutman's Theorem is made in the same way as in [5].

To begin with, by virtue of Lax-Milgram's Theorem one sees that there exists a large enough $\beta>0$ such that $A_{1}+B_{1}+\beta$ Id is invertible, with the inverse operator mapping $H_{s}^{1}(D)$ onto $\left(H_{s}^{1}(D)\right)^{\prime}$. Denote $L_{\beta}=\left(A_{1}+B_{1}+\beta \mathrm{Id}\right)^{-1}, L_{\beta} \in$ $\left.\mathscr{L}\left(\left(H_{s}^{1}(D)\right)^{\prime}, H_{s}^{1}(D)\right)\right)$. Then, for any $g \in\left(H_{s}^{1}(D)\right)^{\prime}$, one has $L_{\beta} u=g$ iff $u$ solves

$$
a_{\beta}(u, v)=\langle g, v\rangle, \quad \forall v \in H_{s}^{1}(D)
$$

where 


$$
\begin{aligned}
a_{\beta}(u, v) & =\alpha \int_{D}\left(\frac{\partial u}{\partial \theta} \frac{\partial v}{\partial \theta} \sin \theta+\frac{1}{\sin \theta} \frac{\partial u}{\partial \phi} \frac{\partial v}{\partial \phi}\right) \mathrm{d} \theta \mathrm{d} \phi \\
& -\int_{D}\left(a_{1} u \frac{\partial v}{\partial \theta} \sin \theta+a_{2} u \frac{\partial v}{\partial \phi}\right) \mathrm{d} \theta \mathrm{d} \phi+\beta \int_{D} u v \sin \theta \mathrm{d} \theta \mathrm{d} \phi
\end{aligned}
$$

We now prove the following result:

Lemma 3.1. Let $g \in L_{s}^{2}(D)$ and $u=L_{\beta}(g)$. Then $u \in H^{1}(\Omega)$ is the unique solution to the problem

$$
\alpha \int_{\Omega} \nabla_{x} u \cdot \nabla_{x} \hat{v} \mathrm{~d} \Omega-\int_{\Omega} \hat{a} u \cdot \nabla_{x} \hat{v} \mathrm{~d} \Omega+\int_{\Omega} \hat{\beta} u \hat{v} \mathrm{~d} \Omega=\int_{\Omega} \hat{g} \hat{v} \mathrm{~d} \Omega, \quad \forall \hat{v} \in H^{1}(\Omega)
$$

where: $\hat{a}: \Omega \rightarrow \mathbb{R}^{3}, \quad \hat{\beta}: \Omega \rightarrow \mathbb{R}$ and $\hat{g}: \Omega \rightarrow \mathbb{R}$ are such that $\hat{a} \cdot e_{r}=0, \hat{a} \cdot e_{\theta}=\frac{a_{1}}{r}, \hat{a} \cdot e_{\phi}=\frac{a_{2}}{r}, \hat{\beta}=\frac{\hat{\beta}}{r^{2}}, \hat{g}=\frac{g}{r^{2}}$.

Proof. Equality (3.7), written in spherical coordinates, looks:

$$
\begin{gathered}
\int_{D} \int_{1}^{2}\left(\frac{1}{r^{2}} \frac{\partial u}{\partial \theta} \frac{\partial \hat{v}}{\partial \theta}+\frac{1}{r^{2} \sin ^{2} \theta} \frac{\partial u}{\partial \phi} \frac{\partial \hat{v}}{\partial \phi}\right) r^{2} \sin \theta \mathrm{d} r \mathrm{~d} \theta \mathrm{d} \phi \\
-\quad \int_{D} \int_{1}^{2} u\left(\frac{1}{r} \hat{a} \cdot e_{\theta} \frac{\partial v}{\partial \theta}+\frac{1}{r \sin \theta} \hat{a} \cdot e_{\phi} \frac{\partial v}{\partial \phi}\right) r^{2} \sin \theta \mathrm{d} r \mathrm{~d} \theta \mathrm{d} \phi \\
+\quad \int_{D} \int_{1}^{2} \hat{\beta} u \hat{v} r^{2} \sin \theta \mathrm{d} \theta \mathrm{d} \phi=\int_{D} \int_{1}^{2} \hat{g} \hat{v} r^{2} \sin \theta \mathrm{d} r \mathrm{~d} \theta \mathrm{d} \phi
\end{gathered}
$$

Therefore, one must show that, for any $\hat{v} \in H^{1}(\Omega)$, the following holds true:

$$
\begin{gathered}
\int_{D} \int_{1}^{2}\left(\frac{\partial u}{\partial \theta} \frac{\partial \hat{v}}{\partial \theta}+\frac{1}{\sin ^{2} \theta} \frac{\partial u}{\partial \phi} \frac{\partial \hat{v}}{\partial \phi}\right) \sin \theta \mathrm{d} \theta \mathrm{d} \phi \\
-\int_{D} \int_{1}^{2} u\left(a_{1} \frac{\partial v}{\partial \theta}+\frac{1}{\sin \theta} a_{2} \frac{\partial v}{\partial \phi}\right) \sin \theta \mathrm{d} \theta \mathrm{d} \phi \\
+\beta \int_{D} \int_{1}^{2} u \hat{v} \sin \theta \mathrm{d} \theta \mathrm{d} \phi=\int_{D} \int_{1}^{2} g \hat{v} \sin \theta \mathrm{d} \theta \mathrm{d} \phi
\end{gathered}
$$

Let $v=\int_{1}^{2} \hat{v} \mathrm{~d} r$ in (3.5), with $\hat{v} \in H^{1}(\Omega)$ arbitrary. It follows that $u$ solves (3.9).

REMARK 3.1. Observe that equality (3.7) is the weak (variational) formulation of the following problem:

$$
\begin{array}{ll}
-\alpha & \triangle_{x} u+\nabla_{x}(\hat{a} u)+\beta u=\hat{g}, \quad \text { on } \quad \Omega \\
-\alpha & \frac{\partial u}{\partial \nu}+\hat{a} \cdot \nu u=0, \quad \text { on } \quad \partial \Omega
\end{array}
$$


Lemma 3.2 (Maximum Principle). Let $f \in L_{s}^{2}(D), u=L_{\beta}(g)$. Then:

(i) whenever $g \geq 0$, we have $u \geq 0$ (weak maximum principle)

(ii) whenever $g \geq 0, g \neq 0$, we have $u>0$ (strong maximum principle)

Proof. (i) it follows immediately by taking $v=u^{-}$in (3.5)

(ii) Denote $D_{0}:=\{(\theta, \phi) \in D, u(\theta, \phi)=0\}$. The proof is by contradiction: assume $D_{0} \neq \varnothing$. Then $D-D_{0} \neq \varnothing$ as well. Let now $\left(\theta_{0}, \phi_{0}\right) \in \partial D_{0}$. Let $x^{0}:=\frac{3}{2}\left(\sin \theta_{0} \cos \phi_{0}, \sin \theta_{0} \sin \phi_{0}, \cos \theta_{0}\right), x^{0} \in \Omega$. One can find an $y^{0} \in \Omega$ and a $r>0$ such that $x^{0} \in \partial B\left(y^{0}, r\right)$, whith $B\left(y^{0}, r\right) \subset\{x \in \Omega, u(x)>0\}$. Since $u$ satisfies the problem (3.10) in $\Omega$, one deduces by the strong maximum principle (see e.g. [6]) that $\frac{\partial u}{\partial \nu_{B}}\left(x^{0}\right)<0$, where $\nu_{B}$ denotes the outward normal to $B\left(y^{0}, r\right)$. On the other hand, since $x^{0}$ is a minimum point for $u$ on $\Omega$ we have $\nabla u\left(x^{0}\right)=0$, a contradiction.

Denote by $C_{\text {per }}(\bar{D})$ the set of continuous functions on $D$ and periodic w.r.t. variable $\phi$, endowed with the usual sup norm. Observe the following continuous inclusion: $C_{\text {per }}(\bar{D}) \hookrightarrow L_{s}^{2}$. One has the following result:

Lemma 3.3 (Compactness). One has $L_{\beta} \in \mathscr{L}\left(C_{\text {per }}(\bar{D}), C_{\text {per }}(\bar{D})\right)$, and $L_{\beta}$ is compact.

Proof. Let $g \in C_{\text {per }}(\bar{D})$ and $u=L_{\beta}(g)$. Then $u$ solves equation (3.7). Clearly $g \in L^{p}(\Omega)$, for any $p>1$, and by the interior smoothness property, it follows that $u \in W^{2, p}\left(\Omega^{\prime}\right)$ for any open set $\Omega^{\prime}$ s.t. $\overline{\Omega^{\prime}} \subset \Omega$.

Next, there exists $c=c\left(\Omega^{\prime}, p\right)$ s.t. $\|u\|_{W^{2, p}\left(\Omega^{\prime}\right)} \leq c\|g\|_{L^{p}(\Omega)} \leq c\|g\|_{C_{\mathrm{per}}(\bar{D})}$, for any $g \in C_{\text {per }}(\bar{D})$. However, the following Sobolev continuous inclusion $W^{2, p}\left(\Omega^{\prime}\right) \hookrightarrow$ $\mathscr{C}^{0}\left(\Omega^{\prime}\right)$ is compact for large enough $p>1$. Then choose an open set $\Omega^{\prime}$ so that it contains the set $\{x \in \Omega, r=3 / 2\}$; the proof is over.

We are now in a position allowing us to state Section 3.1 main result:

THEOREM 3.1. The boundary value problem SP-1 given in equations (3.1)-(3.2) has a unique solution $\psi_{s} \in C_{\text {per }}(\bar{D}), \psi_{s}>0$. Moreover, any eigenvalue $\lambda \in \mathbb{C}$ of $A_{1}+B_{1}$ is s.t. $\operatorname{Re}(\lambda) \geq 0$.

Proof. Let the cone of non-negative functions be defined as $P:=\{u \in$ $\left.C_{\text {per }}(\bar{D}), u \geq 0\right\}$. Clearly its interior is given by $\stackrel{o}{P}:=\left\{u \in C_{\text {per }}(\bar{D}), u>0\right\}$. By Lemma 3.3 and part (ii) in Lemma 3.2 one sees the strong version of KreinRutman's theorem can be applied to operator $L_{\beta}$. Then there exists a simple eigenvalue $\mu>0$ of $L_{\beta}$, and denote $\psi_{s} \in \stackrel{o}{P}$ the corresponding eigenvector. Also observe that $\psi_{s} \in H_{s}^{1}(D)$. Next, $\lambda=\frac{1}{\mu}-\beta$ is a simple eigenvalue of $A_{1}+B_{1}$, and $\left(A_{1}+B_{1}\right) \psi_{s}=\lambda \psi_{s}$. Therefore, $\psi_{s}$ solves, for any $v \in H_{s}^{1}(D)$,:

$$
\begin{aligned}
& \alpha \int_{D}\left(\frac{\partial \psi_{s}}{\partial \theta} \frac{\partial v}{\partial \theta} \sin \theta+\frac{1}{\sin \theta} \frac{\partial \psi_{s}}{\partial \phi} \frac{\partial v}{\partial \phi}\right) \mathrm{d} \theta \mathrm{d} \phi- \\
& \int_{D}\left(a_{1} \psi_{s} \frac{\partial v}{\partial \theta} \sin \theta+a_{2} \psi_{s} \frac{\partial v}{\partial \phi}\right) \mathrm{d} \theta \mathrm{d} \phi=\lambda \int_{D} \psi_{s} v \sin \theta \mathrm{d} \theta \mathrm{d} \phi
\end{aligned}
$$


Let $v=1$ in (3.11); as $\psi_{s}>0$, it results that $\lambda=0$. Therefore $\lambda=0$ is a simple eigenvalue for $A_{1}+B_{1}$, which ends the proof.

REMARK 3.2. One can also prove, using the strong version of the KreinRutman's Theorem as done above, that $\psi_{\text {s }}$ granted by Theorem 3.1 is, up to a multiplicative constant, the unique solution to the problem:

$$
\begin{array}{ll}
-\alpha & \triangle_{x} \psi_{s}+\nabla_{x}\left(\hat{a} \psi_{s}\right)=0, \quad \text { on } \quad \Omega \\
-\alpha & \frac{\partial \psi_{s}}{\partial \nu}+\hat{a} \cdot \nu \psi_{s}=0, \quad \text { on } \quad \partial \Omega
\end{array}
$$

3.2. Existence and uniqueness of solutions for the problem SP-2. We first take on proving the following Poincaré inequality:

Proposition 3.1. There exists $c>0$ such that, for any $v \in H_{s 0}^{1}\left(D_{\sigma}\right)$,

$$
\|v\|_{L_{s}^{2}\left(D_{\sigma}\right)}^{2} \leq c \int_{D_{\sigma}}\left[\left(\frac{\partial v}{\partial \sigma}\right)^{2} \sin \theta+\left(\frac{\partial v}{\partial \theta}\right)^{2} \sin \theta+\frac{1}{\sin \theta}\left(\frac{\partial v}{\partial \phi}\right)^{2}\right] \mathrm{d} \theta \mathrm{d} \phi
$$

ProOf. The above stated result is a straightforward consequence of part (iii) and part (iv) in Proposition 2.1 and of the classical Poincaré inequality:

$$
\|v\|_{L^{2}\left(\Omega_{\sigma}\right)}^{2} \leq c \int_{\Omega_{\sigma}}\left[\left(\frac{\partial v}{\partial \sigma}\right)^{2}+\left(\nabla_{x} v\right)^{2}\right] \mathrm{d} x \mathrm{~d} \sigma, \quad \forall v \in V_{\sigma}
$$

REMARK 3.3. From Proposition 3.1 one infers that the seminorm defined on $H_{s 0}^{1}\left(D_{\sigma}\right)$ by

$$
v \rightarrow \int_{D_{\sigma}}\left[\left(\frac{\partial v}{\partial \sigma}\right)^{2} \sin \theta+\left(\frac{\partial v}{\partial \theta}\right)^{2} \sin \theta+\frac{1}{\sin \theta}\left(\frac{\partial v}{\partial \phi}\right)^{2}\right] \mathrm{d} \theta \mathrm{d} \phi
$$

is equivalent to the usual norm on $H_{s 0}^{1}\left(D_{\sigma}\right)$.

TheOREM 3.2. There exists a unique solution $\overline{\psi_{s}}$ to the problem (3.1)-(3.2).

Proof. By Proposition 3.1, operator $A_{2}$ is invertible; consequently, the problem (3.3) is equivalent to the following one:

$$
\overline{\psi_{s}}+A_{2}^{-1} B_{2} \psi_{s}=-\frac{1}{4 \pi} A_{2}^{-1}\left(B_{2}(1)\right)
$$

Observe $A_{2}^{-1} B_{2}$ is continuous and compact and maps $L_{s}^{2}\left(D_{\sigma}\right)$ onto itself. By Fredholm theory, proving the existence and uniqueness of solutions to (3.16) is tantamount to proving the kernel of $A_{2}+B_{2}$ reduces to $\{0\}$.

Let $u \in H_{s 0}^{1}\left(D_{\sigma}\right)$ be chosen such that $A_{2} u+B_{2} u=0$. Then $u \in V_{\sigma}$ and we have for any $\hat{v} \in V_{\sigma}$ :

$$
\int_{\Omega_{\sigma}}\left(\frac{\alpha_{1}}{r^{2}} \frac{\partial u}{\partial \sigma} \frac{\partial \hat{v}}{\partial \sigma}+\alpha_{2} \nabla_{x} u \cdot \nabla_{x} \hat{v}\right) \mathrm{d} x \mathrm{~d} \sigma-\int_{\Omega_{\sigma}} \hat{a} u \cdot \nabla_{x} \hat{v} \mathrm{~d} x \mathrm{~d} \sigma=0
$$


where $\hat{a}$ is the same as in Lemma 3.1.

Next, we proceed as in $[\mathbf{7}]$. For any $\epsilon>0$, let $v=\frac{u^{+}}{u^{+}+\epsilon}$. Then:

$\nabla_{x, \sigma} v=\epsilon \frac{\nabla_{x, \sigma} u^{+}}{\left(u^{+}+\epsilon\right)^{2}}$

One obtains:

$$
\begin{aligned}
& \int_{\Omega_{\sigma}}\left[\frac{\alpha_{1}}{r^{2}} \frac{1}{\left(u^{+}+\epsilon\right)^{2}}\left(\frac{\partial u^{+}}{\partial \sigma}\right)^{2}+\alpha_{2} \frac{\left(\nabla_{x} u^{+}\right)^{2}}{\left(u^{+}+\epsilon\right)^{2}}\right] \mathrm{d} x \mathrm{~d} \sigma= \\
& \int_{\Omega_{\sigma}} \hat{a} u^{+} \frac{\nabla_{x} u^{+}}{\left(u^{+}+\epsilon\right)^{2}} \mathrm{~d} x \mathrm{~d} \sigma \leq \int_{\Omega_{\sigma}}|\hat{a}|\left|\frac{\nabla_{x} u^{+}}{u^{+}+\epsilon}\right| \mathrm{d} x \mathrm{~d} \sigma \\
& \leq \frac{\alpha_{2}}{2} \int_{\Omega_{\sigma}}\left|\frac{\nabla_{x} u^{+}}{u^{+}+\epsilon}\right|^{2} \mathrm{~d} x \mathrm{~d} \sigma+c
\end{aligned}
$$

Therefore:

$$
\left\|\frac{\nabla_{x} u^{+}}{u^{+}+\epsilon}\right\|_{L^{2}\left(\Omega_{\sigma}\right)} \leq c
$$

where $c$ is a constant which is independent of $\epsilon$. Observe that

$\nabla_{x, \sigma} \log \left(1+\frac{u^{+}}{\epsilon}\right)=\frac{\nabla_{x, \sigma} u^{+}}{u^{+}+\epsilon}$

and that $\log \left(1+\frac{u^{+}}{\epsilon}\right)=0$ whenever $\sigma=0$ and $\sigma=1$. Invoking Poincaré's inequality one gets $\left\|\log \left(1+\frac{u^{+}}{\epsilon}\right)\right\|_{L^{2}\left(\Omega_{\sigma}\right)} \leq c$. The latest, in turn, gives $u^{+}=0$, therefore $u \leq 0$. Reasoning on $u^{-}$instead on $u^{+}$and proceeding likewise leads to $u \geq 0$, which ultimately gives $u=0$. We have thus obtained the existence and uniqueness of $\overline{\psi_{s}} \in L_{s}^{2}\left(D_{\sigma}\right)$, solution to equation (3.16).

Next, let $\psi_{s}=\frac{1}{4 \pi}+\overline{\psi_{s}}$. Then $\psi_{s}$ solves equation (3.17) (with $\psi_{s}$ instead of $u$ ). As $\psi_{s}=\frac{1}{4 \pi}$ for $\sigma=0$ or $\sigma=1$, one has $\psi_{s}^{-} \in V_{\sigma}$. Let now a test function be given by $v=\frac{\psi_{s}^{-}}{\psi_{s}^{-}+\epsilon}$. Proceeding as above, one obtains $\psi_{s}^{-}=0$, therefore $\frac{1}{4 \pi}+\overline{\psi_{s}} \geq 0$.

Now, take any $\varphi=\varphi(\sigma) \in H_{0}^{1}(] 0,1[)$ in the weak formulation given in equation (3.3); it gives $\alpha_{2} \int_{D_{\sigma}} \frac{\partial \overline{\psi_{s}}}{\partial \sigma} \varphi^{\prime}(\sigma) \sin \theta \mathrm{d} \theta \mathrm{d} \phi \mathrm{d} \sigma=0$

Denote $\tilde{\psi}_{s}:=\int_{D_{\sigma}} \overline{\psi_{s}} \sin \theta \mathrm{d} \theta \mathrm{d} \phi$. Then $\tilde{\psi}_{s}^{\prime \prime}(\sigma)=0$. The latest result, together with $\tilde{\psi}_{s}(0)=\tilde{\psi}_{s}(1)=0$, gives $\tilde{\psi}_{s}=0$, ending the proof.

REMARK 3.4. Notice that $\psi_{s}=\overline{\psi_{s}}+\frac{1}{4 \pi}$ is the unique solution to the following problem: find $\psi_{s} \in V_{\sigma}+\frac{1}{4 \pi}$ such that, for all $v \in V_{\sigma}$,

$$
\int_{\Omega_{\sigma}}\left(\frac{\alpha_{1}}{r^{2}} \frac{\partial \psi_{s}}{\partial \sigma} \frac{\partial v}{\partial \sigma}+\alpha_{2} \nabla_{x} \psi_{s} \cdot \nabla_{x} v\right) \mathrm{d} x \mathrm{~d} \sigma-\int_{\Omega_{\sigma}} \frac{\hat{a} \psi_{s}}{r} \cdot \nabla_{x} v \mathrm{~d} x \mathrm{~d} \sigma=0
$$


The above is actually the weak formulation of the problem:

$$
\begin{aligned}
& -\frac{\alpha_{1}}{r^{2}} \frac{\partial^{2} \psi_{s}}{\partial \sigma^{2}}-\alpha_{2} A \psi_{s}+\nabla_{x}\left(\frac{1}{r} \hat{a} \psi_{s}\right)=0, \quad \text { on } \Omega_{\sigma} \\
& -\alpha_{2} \frac{\partial \psi_{s}}{\partial \nu}+\frac{\hat{a} \cdot \nu}{r}=0, \quad \text { on } \quad \partial \Omega_{\sigma} \\
& \left.\psi_{s}\right|_{\sigma=0}=\left.\psi_{s}\right|_{\sigma=1}=\frac{1}{4 \pi}
\end{aligned}
$$

\section{Final comments}

There is growing interest in using phase-space kinetical theories for modeling the thermo-rheological behavior of complex fluids, all the more that, for polymer mixtures and chemically reacting liquids, reptation based approaches seem to be less suitable. In [3] Bird et al exposed at length the physical assumptions and many underpinnings of their polymer dynamics theory.

Crucial to basically any mean field theory is the configurational (probability) density function: it incorporates all inter- and intra-molecular interactions of relevance to a given molecular system. It is thus quite natural to have one's attention focused on ways to solve it. To the best of our knowledge, the question of existence and uniqueness of solutions to this PDE, within the framework of the general phase space theory, has not been answered. In Section 2 we proved that, under physically meaningful initial boundary conditions, the evolutionary PDE has unique solutions.

For large enough times, one is interested in stationary probability density equation solutions. In Section 3 we made use of the Krein-Rutman's Theorem to prove the existence and uniqueness of solutions to the steady-state probability density equation of Bird et al [3] kinetic theory for polymeric systems.

The matter of time depending solutions convergence, for $t \rightarrow+\infty$, towards the stationary ones will be addressed in a subsequent paper.

\section{Acknowledgements}

L.I.P. greatfully acknowledges Professor Robert Byron Bird, University of Wisconsin, Madison, for talks on kinetic theories.

\section{References}

[1] A. N. Beris, B. J. Edwards, Thermodynamics of Flowing Systems With Internal Microstructure, Oxford University Press, 1994.

[2] R. B. Bird, R. C. Armstrong, O. Hassager, Dynamics of Polymeric Liquids, Vol. 1: Fluid Mechanics, J. Wiley \& Sons, New-York, 1987.

[3] R. B. Bird, R. C. Armstrong, O. Hassager, Dynamics of Polymeric Liquids, Vol. 2: Kinetic Theories, J. Wiley \& Sons, New-York, 1987.

[4] J. A. Carillo, S. Cordier, S. Mancini, A decision-making Fokker-Planck model in computational neuroscience, to appear in Journal of Mathematical Biology.

[5] I. S. Ciupercă, L. I. Palade, The steady state configurational distribution diffusion equation of the standard FENE dumbbell polymer model: existence and uniqueness of solutions for arbitrary velocity gradients. Mathematical Models and Methods in Applied Sciences, 19:20392064, 2009.

[6] L.C. Evans, Partial Differential Equations, AMS, Providence, RI, 1998.

[7] G. Gilbarg, N. Trudinger, Elliptic partial differential equations of second order, SpringerVerlag, Berlin, 2001.

[8] R. R. Huilgol, N. Phan-Thien, Fluid Mechanics of Viscoelasticity, Elsevier, 1997.

[9] J. G. Kirkwood, Macromolecules, edited by P. L. Auer, Gordon and Breach, New York, 1968.

[10] R. G. Larson, Constitutive Equations for Polymer Melts and Solutions, Butterworths, 1988. 
[11] F. A. Morrison, Understanding Rheology, Oxford University Press, 2001.

[12] H. C. Öttinger, Beyond Equilibrium Thermodynamics, Wiley, 2006.

[13] M. Renardy, Mathematical Analysis of Viscoelastic Flows, SIAM, 2000.

[14] D. D. Schieber, Generalized Brownian configurations fields for Fokker-Planck equations including center-of-mass diffusion, J. Non-Newt. Fl. Mech. 135:179-181, 2006.

[15] S. Cleja-Ţigoiu, V. Tigoiu, Rheology and Thermodynamics, Part I - Rheology, Editura Universităţii din Bucureşti, 1998.

Université Lyon 1, Institut Camille Jordan UMr5208, Bât Braconnier, 43 BouleVard du 11 Novembre 1918, F-69622, Villeurbanne, France

E-mail address: ciuperca@math.univ-lyon.fr

INSA-Lyon, Institut Camille Jordan UMR5208 \& Pôle de Mathématiques, Bât. Leonard de Vinci No. 401, 21 Avenue Jean Capelle, F-69621, Villeurbanne, France

E-mail address: liviu-iulian.palade@insa-lyon.fr 